\title{
Search for Gluonic Excitations
}

\author{
Paul Eugenio \\ Florida State University, Department of Physics, Tallahassee, Florida 32306 USA
}

\begin{abstract}
Studies of meson spectra via strong decays provide insight regarding QCD at the confinement scale. These studies have led to phenomenological models for QCD such as the constituent quark model. However, QCD allows for a much richer spectrum of meson states which include extra states such as exotics, hybrids, multi-quarks, and glueballs. First discussion of the status of exotic meson searches is given followed by a discussion of plans at Jefferson Lab to double the energy of the machine to $12 \mathrm{GeV}$, which will allow us to access photoproduction of mesons in search for gluonic excited states.
\end{abstract}

Keywords: mesons, hybrids, exotics, gluonic excitations

PACS: $14.40 .-\mathrm{n}, 25.70 . \mathrm{Ef}, 13.25 .-\mathrm{k}$

\section{STATUS OF GLUONIC EXCITITATIONS}

Discoveries of new phenomena in nuclear and particle physics have provided insight into the fundamental constituents of matter. In the past few decades we have seen a new picture emerge in which quarks form the building blocks of nearly all matter. Yet the gluon, which carries the force which binds quarks, can interact with other gluons to form a bound state, or interact as a fundamental constituent of matter along with the quarks. Thus new forms of gluonic or hybrid matter should exist.

Studies of meson spectra via strong decays of hadrons provide insight regarding QCD at the confinement scale. These studies have led to phenomenological models for QCD such as the constituent quark model. However, QCD demands a much richer spectrum of meson states which includes extra states such as hybrids $(q \bar{q} g)$, multiquarks $(q \bar{q} q \bar{q})$, and glueballs ( $g g$ or $g g g$ ). Experiment E852 at Brookhaven National Laboratory is an experiment in meson spectroscopy configured to detect both neutral and charged final states of $\pi^{-} p$ collisions in a search for meson states incompatible with the constituent quark model.

The apparatus is located at the Multi-Particle Spectrometer (MPS) of Brookhaven's Alternating Gradient Synchrotron (AGS). For E852, the AGS delivered an $18 \mathrm{GeV} / \mathrm{c} \pi^{-}$ beam to a fixed liquid hydrogen target at the MPS. The MPS facility was augmented with additional detectors designed specifically for E852 and consists of 3 integral regions: target, charged tracking, and downstream regions.

The target region is located in the middle of the MPS magnet and contains the following elements: a $30.5 \mathrm{~cm}$ long liquid hydrogen target, a four-layer cylindrical driftproportional wire chamber, and a 198 block CsI(Ti) barrel veto detector.

The downstream half of the MPS magnet houses the main components of the charged tracking region. It consists of 3 proportional wire chambers (TPX1-3) and 6 drift chambers modules (DM1-6) each with seven-layers. In addition to the tracking chambers, there are two scintillation veto counters (CPVB and CPVC) and a window-frame lead-

CP947, VII Latin American Symposium on Nuclear Physics and Applications

edited by R. Alarcon, P. L. Cole, C. Djalali, and F. Umeres

(C) 2007 American Institute of Physics 978-0-7354-0461-8/07/\$23.00 
scintillator sandwich veto detector (DEA).

The downstream region contains a 3000 element lead-glass calorimeter (LGD) for detecting and measuring the energy of the forward going gammas and a large drift chamber (TDX4) located directly in front of the LGD for tagging charged particles entering the LGD. A more detailed description of the E852 apparatus is given in Reference [1].

\section{The $\pi_{1}(1400)$ Exotic Meson}

The $\eta \pi$ system is particularly interesting in searching for exotic (or non- $q \bar{q}$ ) mesons because the system has spin $(\mathrm{J})$, parity $(\mathrm{P})$, and charge-conjugation $(\mathrm{C})$ in sequence $J^{P C}=0^{++}, 1^{-+}, 2^{++}, 3^{-+} \ldots$ for $l=0,1,2,3, \ldots$. (Here $l$ is the orbital angular momentum of the $\eta \pi$ system.) Hence a resonance with an $\eta \pi$ decay mode with odd $l$ is manifestly exotic.

In previous publications [2, 3], Brookhaven E852 presented evidence for an exotic meson produced in the reaction $\pi^{-} p \rightarrow \eta \pi^{-} p$ at $18 \mathrm{GeV} / c$ from an analysis of the 1994 E852 data set. A large asymmetry in the angular distribution was observed indicating interference between $l$-even and $l$-odd partial waves. The $a_{2}(1320)$ was observed in the $J^{P C}=2^{++}$wave, as was a broad enhancement between 1200 and $1600 \mathrm{MeV} / \mathrm{c}^{2}$ in the $1^{-+}$exotic wave. The observed phase difference between these waves shows that there was phase motion in addition to that due to $a_{2}(1320)$ decay. An analysis of the mass dependence of the partial waves shows that the data is well described by the interference between the $a_{2}(1320)$ and an exotic $1^{-+}$resonance with $M a s s=\left(1370 \pm 16_{-30}^{+50}\right) \mathrm{MeV} / \mathrm{c}^{2}$ and $\Gamma=\left(385 \pm 40_{-105}^{+65}\right) \mathrm{MeV} / \mathrm{c}^{2}$.

\section{The $\pi_{1}(1600)$ Exotic Meson}

E852 has performed a partial wave analysis of the reaction $\pi^{-} p \rightarrow \pi^{+} \pi^{-} \pi^{-} p$ [4]. In summary, all expected well-known states $\left(a_{1}, a_{2}\right.$, and $\left.\pi_{2}\right)$ are observed. In addition, the natural parity exchange wave with manifestly exotic quantum numbers $J^{P C}=1^{-+}$ shows structure in the intensity and phase motion which is consistent with a resonance at $1600 \mathrm{MeV} / c^{2}$ decaying into the $\rho \pi$ channel. A mass dependent fit results in a resonance mass of $1593 \pm 8_{-47}^{+29} \mathrm{MeV} / c^{2}$ and a width of $168 \pm 20_{-12}^{+150} \mathrm{MeV} / c^{2}$. A recent analysis of a larger $3 \pi$ data set emphasizes discrepancies in the PWA systematics which make the claims of an exotic signal controversial [5].

A subsequent analysis studied the $\eta^{\prime} \pi^{-}$system produced in the reaction $\pi^{-} p \rightarrow$ $\eta \pi^{+} \pi^{-} \pi^{-} p$. Figure 1 a shows a clear signal for $\eta^{\prime} \rightarrow \eta \pi^{+} \pi^{-}$in the $\eta \pi^{+} \pi^{-}$mass spectrum, and shown in Figure $1 \mathrm{~b}$ is the accepted $\eta^{\prime} \pi^{-}$mass spectrum which exhibits a peak in the region of the $a_{2}(1320)$ and a broad peak near $1600 \mathrm{MeV} / c^{2}$. The results of a partial wave analysis find two important partial waves: the $2^{++}$wave consistent with an $a_{2}(1320)$ signal and a broad higher mass structure; and a dominant $J^{P C}=1^{-+}$exotic wave which peaks at $1600 \mathrm{MeV} / \mathrm{c}^{2}$.

A study of the mass dependence of the PWA results finds three Breit-Wigner (BW) poles in the $2^{++}$wave and two BW poles in the $1^{-+}$wave. Figure 2 displays the 

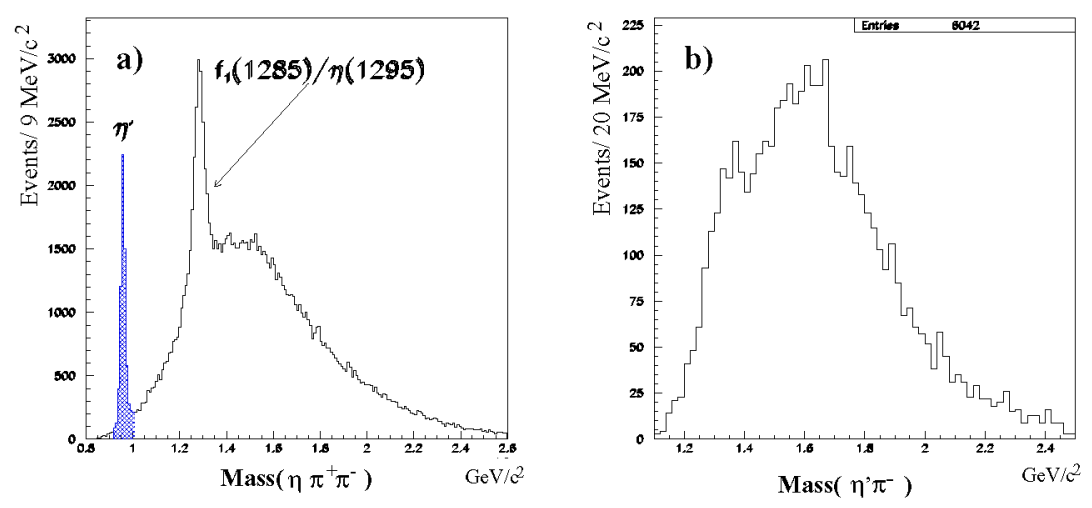

FIGURE 1. a) The accepted $\eta \pi^{+} \pi^{-}$effective mass distribution. b) The accepted $\eta^{\prime} \pi^{-}$effective mass distribution.

PWA results (data points with errors) with the curves showing the results of the mass dependent fit.

Since the $1^{-+}$wave is dominated by a pole at $1600 \mathrm{MeV} / c^{2}$ with a small contribution from a pole at $1400 \mathrm{MeV} / \mathrm{c}^{2}$, an alternative fit, one with a poorer $\chi^{2} / D o F$ of 1.22 , finds that the $1^{-+}$wave can be described by the pole at $1600 \mathrm{MeV} / \mathrm{c}^{2}$ only. The results for the $2^{++}$poles and the $1^{-+}$pole at $1600 \mathrm{MeV} / \mathrm{c}^{2}$ are stable and are not affected by the parameterization of the $1^{-+}$low mass region.

In the Fux-tube model the lightest 1-+ isovector hybrid is predicted to decay primarily to $b_{1} \pi[11,12,13]$. The $f_{1} \pi$ branch is also expected to be large and many other decay modes are suppressed. This suppression is consistent with recent calculations showing $1 / \mathrm{Nc}$ behavior for decays to spin-zero mesons in the large-Nc limit of QCD [6].

Few experiments have addressed the $b_{1} \pi$ and $f_{1} \pi$ meson decay channels. The VES collaboration reported a broad $J^{P C}=1^{-+}$peak in $b_{1} \pi$ decay [7], and Lee, et al. [8] observed significant $J^{P C}=1^{-+}$signal in a $f_{1} \pi$ decay. In neither case was a definitive resonance interpretation of the 1-+ waves possible. Preliminary results from a later VES analysis show excitation of $\pi_{1}(1600)$ [9]. Significant $b_{1} \pi$ strength for $\pi_{1}(1600)$ was also reported [10]. Recently BNL experiment E852 reported a measurement of $f_{1} \pi$ and $b_{1} \pi$ decays for $\pi_{1}(1600)$ and $\pi_{1}(2000)[11,12]$.

\section{GLUEX: THE SCIENCE OF QUARK CONFINEMENT \& GLUONIC EXCITATIONS}

A recent effort at Jefferson Lab, in conjunction with the plans for the energy upgrade of CEBAF, is the development of a state-of-the-art hermetic spectrometer-the GlueX project (formerly know as the Hall D project). One of the scientific motivations for upgrading CEBAF is a high-statistics definitive study of the photoproduction of mesons with masses below the $c \bar{c}$ threshold in a search for new forms of hadronic and gluonic 

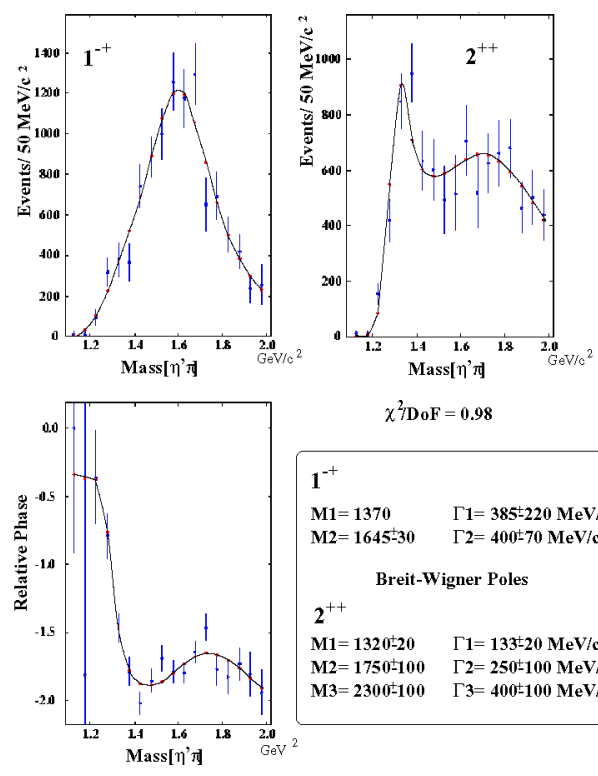

$\chi^{2} \mathrm{DOOF}=0.98$

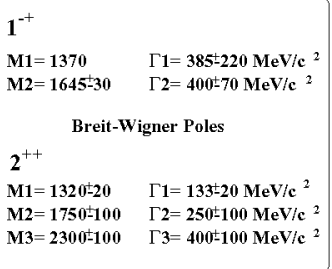

FIGURE 2. The mass dependency of the partial wave intensities and relative phases. Results of the PWA are given as data points with errors whereas results of a mass dependent fit assuming 2 poles in the $1^{-+}$and 3 poles in the $2^{++}$are shown as curves.

matter. After many years of searching for gluonic excitation produced via hadronic probes, only now are we finding some promising candidates. An unexplored search area is the photoproduction of light quark meson states. Since the photon can be thought of as a virtual vector meson with quark spins aligned, it is a probe distinct from the traditional hadronic probes of pions, kaons, and protons. Within the Flux-tube model, the production of gluonic excitations via photon interaction is expected to produce a wealth of states with manifestly exotic $J^{P C}$, s ( $q \bar{q}$ forbidden quantum numbers). The goal of the GlueX experiment is a mapping of the spectrum of gluonic excitations with the ultimate objective being a quantitative understanding of the nature of confinement in QCD. To achieve this goal a hermetic detector, the GlueX spectrometer, optimized for amplitude analysis, will be constructed in a new experimental hall (Hall D). A tagger facility will produce $9 \mathrm{GeV}$ linearly polarized photons via coherent bremsstrahlung radiation of $12 \mathrm{GeV}$ electrons through a diamond wafer. To achieve $12 \mathrm{GeV}$ electrons CEBAF will be upgraded to $12 \mathrm{GeV}$ with additional cryomodules, modified arcs and an additional arc. Critical Decision 0 (CD-0) for the upgrade and GlueX was awarded by the Department of Energy (DOE) in 2004 followed by Critical Decision 1 (CD-1) approval in 2006. The GlueX collaboration was formed in 1998. The project has been reviewed externally and by the Jefferson Lab PAC. The GlueX management has been in place since 2000 with a Spokesman, Deputy-spokesman, Hall D group leader and an elected Collaboration 
Board.

\section{HYCLAS: THE SEARCH FOR GLUONIC HYBRID MESONS WITH THE JLAB CLAS FACILITY}

One of Jefferson Lab's most important missions is the study of QCD at intermedate energies. Meson spectroscopy is one of the leading ways to study QCD at the confinement scale. Exotics, Hybrids, and Strangeonia are poorly known, and they represent the next frontier in hadronic physics. The spectroscopy of mesons in the 1 to $2 \mathrm{GeV} / \mathrm{c}^{2}$ mass range will provide insight into these new forms of hadronic matter, and thus aid in the study of QCD at the realm of confinement. Tantalizing evidence for "gluonic hybrid" states exists, but the interpretation is controversial. Future projects such as GlueX at Jefferson Lab and PANDA at GSI will eventually provide definitive measurements of exotic states, but it will be several years before these experiments will acquire data. Jefferson Lab offers an unique opportunity to undertake the study of meson spectroscopy at intermediate energies. And current studies at CLAS are showing the feasibility of using CLAS as a meson spectrometer for few-body final states.

Motivated by these and recent experimental results for gluonic hybrid candidates and from recent theoretical Lattice QCD and Flux-tube model calculations, Paul Eugenio (as spokesperson/contact person for JLAB E04-005) led the effort to develop a program for a high statistical search for gluonic hybrid mesons and strangeonia ${ }^{1}$ utilizing a high energy photon beam at CLAS. Given the current limited acceptance of CLAS, E04-005 will concentrate on important final states but with the focus on channels with a limited number of three to four charged particles in the final state.

The Jefferson Laboratory Program Advisory Committee (JLAB PAC 25) held its 25th meeting on January $12-18,2004$. The PAC, in response to the charge from the JLab Director, Dr. Christoph Leemann, reviewed and made recommendations for proposals and letters of intent submitted by Jefferson Lab users. Our proposal (E04-005) was awarded 35 days of beam-time received an "A" approval rating. In its report, the PAC stated,

In a measurement in Hall B, the search for new forms of hadronic matter in the meson sector will complement the ongoing investigations in the baryon sector. The chances of identifying exotic meson states with this experiment look very promising. The progress made by the proposers in establishing phase shift analysis procedures in the analysis of CLAS data has been remarkable. This is an experiment of exceptional promise, putting Jefferson Laboratory at the forefront of exotic meson spectroscopy.

The program will perform a high statistical partial wave analysis of meson spectroscopy data acquired using CLAS. The program will study meson systems in the mass ranging from 1.0 to $2.3 \mathrm{GeV}$ in reactions having three to four charged particles in the

\footnotetext{
${ }^{1}$ mesonic states with hidden strange quark content
} 
final state of the form:

$$
\begin{gathered}
\gamma p \rightarrow p \pi^{+} \pi^{-} \pi^{0} \\
\gamma p \rightarrow n \pi^{+} \pi^{+} \pi^{-} \\
\gamma p \rightarrow p \phi \eta \rightarrow p K^{+} K^{-} \eta \\
\gamma p \rightarrow n \phi \pi^{+} \rightarrow n K^{+} K^{-} \pi^{+} \\
\gamma p \rightarrow n \pi^{+} \pi^{-} \pi^{+} \pi^{-} \\
\gamma p \rightarrow \Delta^{++} \eta \pi^{-} \rightarrow p \pi^{+} \pi^{-} \eta \\
\gamma p \rightarrow \Delta^{++} \omega \pi^{-} \rightarrow p \pi^{+} \omega \pi^{-} \\
\gamma p \rightarrow n \eta / \pi^{+} \rightarrow n \pi^{+} \pi^{-} \eta \pi^{+} \\
\gamma p \rightarrow p K^{ \pm} K^{0} \pi^{\mp} \\
\gamma p \rightarrow p p \bar{p}
\end{gathered}
$$

The photon energy required to reach the production threshold for a meson $\mathrm{X}$ of mass $m_{X}$ is ( $M_{t}$ is the proton target mass and $M_{r}$ is the baryon recoil mass)

$$
E_{\gamma}=\frac{m_{X}^{2}+M_{r}^{2}-M_{t}^{2}+2 m_{X} M_{r}}{2 M_{t}}
$$

Therefore, with a 5-6 GeV photon beam we can reach up to $2.3-2.5 \mathrm{GeV}$ in meson masses. However, since we need to populate enough phase space for the decay products of the mesons to be experimentally measurable, at least an extra $0.5 \mathrm{GeV}$ from the threshold energy is required. For example, for the $\phi(1850)$ decays to be detected, it will be necessary for photon energies of around $5 \mathrm{GeV}$. Higher beam energies are desirable to isolate the baryon and meson decays in phase space. However, in the case of strangeness production, the number of strange baryons contributing to the background are less and better isolated than for $n \bar{n}$ backgrounds. In some cases, i.e., $K \bar{K} \pi$ decay channel, baryon backgrounds are not present.

\section{REFERENCES}

1. P. Eugenio et al., Phys. Lett. B497, 190 (2001).

2. D. R. Thompson et al. (BNL-E852 Collaboration), Phys. Rev. Lett. 79, 1630 (1997).

3. S. U. Chung et al.(BNL-E852 Collaboration), Phys. Rev.D65, 072001, (1999).

4. G. S. Adams et al. (BNL-E852 Collaboration), Phys. Rev. Lett. 81, 5760, (1998).

5. A. R. Dzierba et al., Phys. Rev. D73, 072001, (2006).

6. Philip R. Page, Phys. Rev. D70, 016004, (2004).

7. Valery Dorofeev et al., AIP Conf. Proc. 619, 143, (2002).

8. J.H. Lee et al., Phys. Lett. B323, 227, (1994).

9. D.V. Amelin, et al., Yad. Fiz. 62, 487 (1999); ibid, Phys. Atom. Nucl. 62, 445, (1999).

10. C.A. Baker et al., Phys. Lett. B563, 140, (2003).

11. M. Lu et al. (BNL-E852 Collaboration), Phys. Rev. Lett. 94, 032002, (2005).

12. J. Kuhn et al. (BNL-E852 Collaboration), Phys. Lett.B595, 109 (2004). 